\title{
Optical polarization of 47 quasi-stellar objects: The data ${ }^{\star \star \star \star}$
}

\author{
H. Lamy and D. Hutsemékers ${ }^{\star \star \star}$ \\ Institut d'Astrophysique, Université de Liège, 5 Av. de Cointe, B-4000 Liège, Belgium \\ Received October 18, 1999; accepted January 3, 2000
}

\begin{abstract}
New broad-band linear polarization measurements are presented for a sample of 47 QSOs which includes 27 broad absorption line QSOs and 2 gravitational lens candidates.
\end{abstract}

Key words: quasars: general — quasars: absorption lines - gravitational lensing — polarization

\section{Introduction}

Since the systematic surveys of Moore \& Stockman (1981, 1984), the optical linear polarization has been recognized as an important quantity for understanding the nature of quasi-stellar objects (QSOs), namely due to the fact that polarization is an indicator of departures from spherical symmetry. This is particularly true for the broad absorption line (BAL) QSOs which appear systematically more polarized than other radio-quiet QSOs.

The new data presented here have been obtained to complement our recent study of BAL QSO polarization (Hutsemékers et al. 1998), as well as to further investigate the discovery of large-scale coherent orientations in the distribution of quasar polarization vectors (Hutsemékers 1998). While the results of these investigations will be published elsewhere (Hutsemékers \& Lamy, in preparation), the present paper provides the full set of new polarimetric data, with details on the observations, the data reduction, and the measurements.

\section{The observations}

The polarimetric observations were carried out during several runs at the European Southern Observatory (ESO La

\footnotetext{
* Tables 3 and 4 are also available in electronic form at the CDS via anonymous ftp to cdsarc.u-strasbg.fr (130.79.128.5) or via http://cdsweb.u-strasbg.fr/Abstract.html

$\star \star$ Based on observations collected at the European Southern Observatory (ESO, La Silla).

$\star \star \star$ Also, Chercheur Qualifié au Fonds National de la Recherche Scientifique (FNRS, Belgium).
}

Silla, Chile) from 1996 to 1999, using the $3.6 \mathrm{~m}$ telescope equipped with the EFOSC camera and spectrograph. In 1996, the instrument was EFOSC1 and the detector a $512 \times 512$ TeK CCD (ESO\#26) with a pixel size of $27 \mu \mathrm{m}$ corresponding to $0^{\prime \prime} 605$ on the sky (Melnick et al. 1989). Later, EFOSC1 was replaced by EFOSC2, and the detector was a $2048 \times 2048$ Loral/Lesser CCD (ESO\#40) with a pixel size of $15 \mu \mathrm{m}$ corresponding to 0 "' 157 on the sky (Patat 1999). The latter CCD was used in a $2 \times 2$ binning mode, except in October 98.

With EFOSC, polarimetry is performed by inserting in the parallel beam a Wollaston prism which splits the incoming light rays into two orthogonally polarized beams. Each object in the field has therefore two images on the CCD detector, separated by $10^{\prime \prime}$ or $20^{\prime \prime}$ (depending on the Wollaston), and orthogonally polarized. To avoid image overlapping, one puts at the telescope focal plane a special mask made of alternating transparent and opaque parallel strips whose width corresponds to the splitting. The object is positioned at the centre of a transparent strip which is imaged on a region of the CCD chosen as clean as possible. The final CCD image then consists of alternate orthogonally polarized strips of the sky, two of them containing the polarized images of the object itself (di Serego Alighieri 1989, 1998; Lamy \& Hutsemékers 1999). Note that the polarization measurements do not depend on variable transparency or seeing since the two orthogonally polarized images of the object are simultaneously recorded. The $20^{\prime \prime}$ Wollaston was used during the September 96, April 98, and September 99 observing runs, while the $10^{\prime \prime}$ Wollaston was used in October 98 and April 99.

In order to derive linear polarization measurements, i.e. the two normalized Stokes parameters $q$ and $u$, frames must be obtained with at least two different orientations of the Wollaston. With EFOSC1, this was done by rotating the whole instrument by $45^{\circ}$ (usually at the adapter angles $270^{\circ}$ and $225^{\circ}$ ) such that, for each object, two frames were secured (Melnick et al. 1989). The excellent transmission of the Wollaston makes these two 
orientations sufficient (di Serego Alighieri 1989). With EFOSC2, a half-wave plate (HWP) is inserted in the optical path and four frames with the HWP at position angles $0^{\circ}, 22.5^{\circ}, 45^{\circ}$, and $67.5^{\circ}$ were obtained, without the need of rotating the whole instrument (Schwarz \& Guisard 1995).

Most observations were done with the Bessel $V$ filter. A few additional ones were obtained with the Gunn $i$ filter. Typical exposure times are around 5 minutes per frame. Seeing was around 1".2 except in September 99 $\left(\sim 5^{\prime \prime}\right)$. In addition, polarimetric calibration stars were observed in the same filters in order to unambiguously fix the zero-point of the polarization position angle and to check the whole observing and reduction process. The observed standard stars are given in Table 1.

\section{Data reduction}

Considering first the two frames obtained with the EFOSC1 rotated at $270^{\circ}$ and $225^{\circ}$, the normalized Stokes parameters $q$ and $u$ are given by

$q=\frac{I_{270}^{\mathrm{u}}-I_{270}^{1}}{I_{270}^{\mathrm{u}}+I_{270}^{1}} \quad$ and $\quad u=\frac{I_{225}^{\mathrm{u}}-I_{225}^{1}}{I_{225}^{\mathrm{u}}+I_{225}^{1}}$,

where $I^{\text {u }}$ and $I^{1}$ respectively refer to the intensities integrated over the upper and lower orthogonally polarized images of the object.

When the four frames with the HWP oriented at $0^{\circ}$, $22.5^{\circ}, 45^{\circ}$ and $67.5^{\circ}$ are considered, the normalized Stokes parameters are derived using the following formulae:

$q=\frac{R_{q}-1}{R_{q}+1} \quad$ where $\quad R_{q}^{2}=\frac{I_{0}^{\mathrm{u}} / I_{0}^{1}}{I_{45}^{\mathrm{u}} / I_{45}^{1}}$,

$u=\frac{R_{u}-1}{R_{u}+1} \quad$ where $\quad R_{u}^{2}=\frac{I_{22.5}^{\mathrm{u}} / I_{22.5}^{1}}{I_{67.5}^{\mathrm{u}} / I_{67.5}^{1}}$,

$I_{\beta}^{\mathrm{u}}$ and $I_{\beta}^{1}$ respectively denoting the intensities integrated over the upper and the lower images of the object, $\beta$ representing the position angle of the HWP. This combination of four frames obtained with different HWP orientations not only removes most of the instrumental polarization (di Serego Alighieri 1998¹), but is essential for correcting the effects of image distortions introduced by the HWP (Lamy \& Hutsemékers 1999). Note that $q$ and $u$ are measured with respect to the instrumental reference frame.

It is clear from these relations that intensities must be determined with the highest accuracy. For this, the data were first corrected for bias and dark emission, and flat-fielded. A plane was locally fitted to the sky around

\footnotetext{
1 We confirm the low intrumental polarization measured by di Serego Alighieri (1998). Indeed, on 27 April 1998, we have observed an unpolarized standard star (HD 154892, from Turnshek et al. 1990) for which we measured $p_{\mathrm{V}}=0.06 \pm$ $0.02 \%$, using Eq. (2).
}

Table 1. Polarized calibration stars

\begin{tabular}{lll}
\hline Date & Object & Ref \\
\hline $11-09-96$ & HD 161291 & 1 \\
$27-04-98$ & HD 111579, HD 155197, HD 298383 & 2,3 \\
$28-04-98$ & HD 155197 & 2,3 \\
$18-10-98$ & HD 298383 & 2 \\
$13-04-99$ & HD 164740, HD 126593, HD 161291, HD 298383 & 1,2 \\
$14-04-99$ & HD 111579, HD 126593, HD 161291, HD 298383 & 1,2 \\
$07-09-99$ & HD 283812 & 2,4 \\
\hline
\end{tabular}

References: (1): Schwarz 1987; (2) Turnshek et al. 1990; (3) Schmidt et al. 1992; (4) Whittet et al. 1992.

Table 2. Residual instrumental polarization

\begin{tabular}{lllll}
\hline Date & $\bar{q}_{\star}$ & $\begin{array}{l}\bar{u}_{\star} \\
(\%)\end{array}$ & $\begin{array}{l}\bar{\sigma}_{\star} \\
(\%)\end{array}$ & $n_{\star}$ \\
\hline $04 / 98$ & -0.07 & +0.01 & 0.17 & 15 \\
$04 / 99$ & +0.00 & +0.19 & 0.17 & 16 \\
\hline
\end{tabular}

each object image, and subtracted from each image individually. Since it appeared that standard aperture photometry was not accurate enough, we have measured the object center at subpixel precision by fitting a $2 \mathrm{D}$ Gaussian profile and integrated the flux in a circle of same center and arbitrary radius by taking into account only those fractions of pixels inside the circle. With this method, the Stokes parameters may be computed for any reasonable radius of the aperture circle. They were found to be stable against radius variation, giving confidence in the method even when the object images are distorted (Lamy \& Hutsemékers 1999). In order to take as much flux as possible with not too much sky background, we fixed the aperture radius at $\alpha \times\left[(2 \ln 2)^{-1 / 2} \mathrm{HWHM}\right]$, where $\alpha=2.5$ with EFOSC1 and $\alpha=3.0$ with EFOSC2 to account for the image elongation introduced by the HWP. HWHM represents the mean half-width at half-maximum of the Gaussian profile. Note that in the few cases where the objects are resolved into multiple components, we use the smallest rectangular aperture encompassing all the components. The whole procedure has been implemented within the ESO MIDAS reduction package.

First applied to the calibration stars, the method provides polarization degrees in excellent agreement with the published values. During some nights more than one star has been observed (Table 1) in order to check the stability and the reliability of the zero-point offset of the polarization position angle. For all stars observed during a given night, the values of the angle offset do agree within $1^{\circ}$, which is quite small given the fact that the EFOSC2 HWP is not achromatic.

The normalized Stokes parameters $q$ and $u$ were then computed for the QSO sample, and modified according to the zero-point offset determined for each night independently. The uncertainties $\sigma_{q}$ and $\sigma_{u}$ are evaluated by 
Table 3. Polarimetric measurements

\begin{tabular}{|c|c|c|c|c|c|c|c|c|c|}
\hline Object & $z$ & Date & Filter & $\begin{array}{r}q \\
(\%)\end{array}$ & $\begin{array}{r}u \\
(\%)\end{array}$ & $\begin{array}{r}\sigma \\
(\%)\end{array}$ & $\begin{array}{r}q_{\star} \\
(\%)\end{array}$ & $\begin{array}{r}u_{\star} \\
(\%)\end{array}$ & $\begin{array}{r}\sigma_{\star} \\
(\%)\end{array}$ \\
\hline B0059-2735 & 1.59 & $11-09-96$ & $V$ & 1.38 & -0.45 & 0.15 & - & - & - \\
\hline B0059-2735* & 1.59 & $11-09-96$ & $i$ & 2.06 & -1.14 & 0.17 & - & - & - \\
\hline B $0846+1540^{\star}$ & 2.91 & 14-04-99 & $V$ & 0.43 & -0.48 & 0.12 & - & - & - \\
\hline B0856+1714* & 2.32 & 14-04-99 & $V$ & 0.70 & 0.20 & 0.17 & 0.10 & 0.13 & 0.03 \\
\hline $\mathrm{B} 1009+0222^{\star}$ & 1.35 & 13-04-99 & $V$ & 0.06 & -0.58 & 0.08 & 0.07 & 0.26 & 0.07 \\
\hline $\mathrm{J} 1053-0058^{\star}$ & 1.55 & 13-04-99 & $V$ & -1.90 & 0.16 & 0.08 & - & - & - \\
\hline $\mathrm{J} 1104-0004^{\star}$ & 1.35 & 13-04-99 & $V$ & 0.49 & 0.14 & 0.13 & 0.16 & -0.02 & 0.05 \\
\hline $\mathrm{J} 1141-0141^{\star}$ & 1.27 & 13-04-99 & $V$ & -0.10 & 0.56 & 0.16 & 0.14 & 0.01 & 0.08 \\
\hline B1151+1145 & 0.18 & $28-04-98$ & $V$ & -0.74 & -0.24 & 0.06 & -0.14 & -0.24 & 0.04 \\
\hline $\mathrm{B} 1157-2354^{\star}$ & 2.10 & $27-04-98$ & $V$ & -1.38 & -0.24 & 0.04 & 0.00 & 0.04 & 0.04 \\
\hline B1157-2354* & 2.10 & 14-04-99 & $V$ & -1.33 & -0.38 & 0.05 & -0.03 & 0.18 & 0.05 \\
\hline B1157+0128 & 1.99 & $13-04-99$ & $V$ & 0.16 & 0.93 & 0.07 & 0.21 & -0.09 & 0.10 \\
\hline B1158+0045 & 1.38 & 13-04-99 & $V$ & -0.37 & 0.42 & 0.10 & -0.16 & 0.10 & 0.04 \\
\hline $\mathrm{B} 1203+1530^{\star}$ & 1.63 & 13-04-99 & $V$ & 0.75 & 1.54 & 0.10 & 0.18 & -0.02 & 0.17 \\
\hline $\mathrm{B} 1205+1436^{\star}$ & 1.64 & 27-04-98 & $V$ & 0.58 & -0.51 & 0.07 & - & - & - \\
\hline B1210+1942 & 1.24 & 13-04-99 & $V$ & -0.29 & 0.34 & 0.08 & -0.19 & 0.35 & 0.11 \\
\hline $\mathrm{B} 1215+1244^{\star}$ & 2.08 & 28-04-98 & $V$ & 0.45 & 0.35 & 0.17 & - & - & - \\
\hline $\mathrm{B} 1216+1103^{\star}$ & 1.62 & $27-04-98$ & $V$ & -0.41 & 0.48 & 0.09 & -0.85 & 0.09 & 0.24 \\
\hline $\mathrm{B} 1219+1244^{\star}$ & 1.31 & $27-04-98$ & $V$ & 0.29 & -0.57 & 0.10 & -0.32 & -0.24 & 0.11 \\
\hline B1222+1437 & 1.55 & $27-04-98$ & $V$ & -0.22 & 0.19 & 0.06 & -0.08 & -0.04 & 0.05 \\
\hline $\mathrm{J} 1225-0150^{\star}$ & 2.04 & 14-04-99 & $V$ & -0.43 & -0.48 & 0.14 & -0.12 & 0.27 & 0.05 \\
\hline $\mathrm{B} 1228+1216^{\star}$ & 1.41 & 27-04-98 & $V$ & -0.04 & -0.11 & 0.06 & 0.21 & 0.48 & 0.22 \\
\hline $\mathrm{B} 1230+1705^{\star}$ & 1.42 & 27-04-98 & $V$ & -0.35 & -0.10 & 0.09 & -0.09 & 0.24 & 0.06 \\
\hline B1230-2347 & 1.84 & $28-04-98$ & $V$ & -0.11 & 0.04 & 0.08 & - & - & - \\
\hline B1234-0209 & 1.62 & 27-04-98 & $V$ & -0.51 & 0.32 & 0.07 & -0.30 & 0.33 & 0.10 \\
\hline B1235-1813 & 2.19 & 13-04-99 & $V$ & 0.97 & -0.14 & 0.05 & - & - & - \\
\hline $\mathrm{B} 1235+1807^{\star}$ & 0.45 & 14-04-99 & $V$ & 0.05 & 0.29 & 0.17 & 0.17 & 0.42 & 0.03 \\
\hline B1238-0944 & 2.09 & 28-04-98 & $V$ & -0.24 & 0.07 & 0.06 & - & - & - \\
\hline $\mathrm{B} 1239+0955^{\star}$ & 2.01 & $27-04-98$ & $V$ & 0.58 & -0.49 & 0.06 & 0.03 & -0.12 & 0.03 \\
\hline B1239+1435 & 1.95 & $28-04-98$ & $V$ & 0.06 & 0.13 & 0.10 & 0.21 & 0.58 & 0.20 \\
\hline B1242+0006 & 2.08 & 28-04-98 & $V$ & -0.15 & 0.21 & 0.08 & -0.10 & 0.05 & 0.07 \\
\hline $\mathrm{J} 1252+0053^{\star}$ & 1.69 & 14-04-99 & $V$ & -0.02 & -0.02 & 0.06 & - & - & - \\
\hline B1256-1734 & 2.06 & $27-04-98$ & $V$ & -0.78 & 0.58 & 0.08 & 0.28 & 0.23 & 0.08 \\
\hline B1258-1627 & 1.71 & 28-04-98 & $V$ & -0.13 & -0.52 & 0.07 & -0.13 & -0.05 & 0.04 \\
\hline B1305+0011 & 2.11 & $27-04-98$ & $V$ & 0.30 & -0.58 & 0.14 & 0.05 & 0.33 & 0.06 \\
\hline $\mathrm{B} 1333+2840^{\star}$ & 1.91 & 13-04-99 & $V$ & 4.66 & -3.39 & 0.11 & -0.15 & 0.31 & 0.24 \\
\hline $\mathrm{B} 1334+2614^{\star}$ & 1.88 & 13-04-99 & $V$ & -0.14 & 0.01 & 0.08 & - & - & - \\
\hline B1416-1256 & 0.13 & $28-04-98$ & $V$ & -0.21 & 0.35 & 0.08 & -0.49 & 0.54 & 0.07 \\
\hline B1429-0053 & 2.08 & 13-04-99 & $V$ & 0.29 & 0.37 & 0.09 & -0.36 & 0.16 & 0.06 \\
\hline B1429-0036 & 1.18 & 14-04-99 & $V$ & -0.06 & 0.15 & 0.10 & - & - & - \\
\hline $\mathrm{B} 1443+0141^{\star}$ & 2.45 & 13-04-99 & $V$ & 1.00 & -0.68 & 0.15 & -0.60 & -0.13 & 0.10 \\
\hline B1451-3735 & 0.31 & 14-04-99 & $V$ & 0.11 & -0.05 & 0.05 & -0.23 & 0.16 & 0.06 \\
\hline B1500+0824 & 3.94 & 14-04-99 & $V$ & -1.09 & -0.19 & 0.28 & -0.26 & 0.16 & 0.12 \\
\hline B2118-4303* & 2.20 & $28-04-98$ & $V$ & -0.11 & -0.65 & 0.11 & - & - & - \\
\hline B2149-2745* & 2.03 & $18-10-98$ & $V$ & -0.13 & 0.07 & 0.10 & 0.00 & 0.25 & 0.15 \\
\hline B2226-3905 & 1.13 & 07-09-99 & $V$ & -0.13 & 0.16 & 0.09 & - & - & - \\
\hline B2240-3702* & 1.83 & $11-09-96$ & $V$ & 1.16 & 1.75 & 0.08 & - & - & - \\
\hline B2240-3702* & 1.83 & $11-09-96$ & $i$ & 1.33 & 0.73 & 0.10 & - & - & - \\
\hline B2329-3828 & 1.19 & 07-09-99 & $V$ & -0.12 & -0.42 & 0.08 & - & - & - \\
\hline B2341-2333* & 2.82 & $18-10-98$ & $V$ & -0.28 & -0.58 & 0.11 & -0.03 & 0.39 & 0.13 \\
\hline
\end{tabular}


Table 4. Final polarimetric data

\begin{tabular}{|c|c|c|c|c|c|c|c|c|}
\hline Object & $\begin{array}{r}q \\
(\%)\end{array}$ & $\begin{array}{r}u \\
(\%)\end{array}$ & $\begin{array}{r}p \\
(\%)\end{array}$ & $\begin{array}{r}\sigma_{p} \\
(\%)\end{array}$ & $\begin{array}{r}p_{0} \\
(\%)\end{array}$ & $\begin{array}{r}p_{\text {ISM }} \\
(\%)\end{array}$ & $\begin{array}{r}\theta \\
\left(^{\circ}\right)\end{array}$ & $\begin{array}{r}\sigma_{\theta} \\
\left(^{\circ}\right)\end{array}$ \\
\hline B0059-2735* & 1.38 & -0.45 & 1.45 & 0.23 & 1.43 & 0.16 & 171 & 5 \\
\hline B0059-2735* & 2.06 & -1.14 & 2.35 & 0.24 & 2.34 & 0.16 & 166 & 3 \\
\hline B $0846+1540^{\star}$ & 0.43 & -0.67 & 0.80 & 0.21 & 0.77 & 0.17 & 151 & 8 \\
\hline $\mathrm{B} 0856+1714^{\star}$ & 0.70 & 0.01 & 0.70 & 0.24 & 0.66 & 0.09 & 0 & 10 \\
\hline $\mathrm{B} 1009+0222^{\star}$ & 0.06 & -0.77 & 0.77 & 0.19 & 0.75 & 0.06 & 137 & 7 \\
\hline J $1053-0058^{\star}$ & -1.90 & -0.03 & 1.90 & 0.19 & 1.89 & 0.17 & 90 & 3 \\
\hline J $1104-0004^{\star}$ & 0.49 & -0.05 & 0.49 & 0.21 & 0.45 & 0.12 & 177 & 13 \\
\hline J $1141-0141^{\star}$ & -0.10 & 0.37 & 0.38 & 0.23 & 0.32 & 0.06 & 53 & 21 \\
\hline $\mathrm{B} 1151+1145$ & -0.67 & -0.25 & 0.72 & 0.18 & 0.70 & 0.01 & 100 & 7 \\
\hline $\mathrm{B} 1157-2354^{\star}$ & -1.31 & -0.25 & 1.33 & 0.17 & 1.32 & 0.44 & 95 & 4 \\
\hline $\mathrm{B} 1157-2354^{\star}$ & -1.33 & -0.57 & 1.45 & 0.18 & 1.44 & 0.44 & 102 & 4 \\
\hline B1157+0128 & 0.16 & 0.74 & 0.76 & 0.18 & 0.74 & 0.01 & 39 & 7 \\
\hline B1158+0045 & -0.37 & 0.23 & 0.44 & 0.20 & 0.40 & 0.01 & 74 & 14 \\
\hline $\mathrm{B} 1203+1530^{\star}$ & 0.75 & 1.35 & 1.54 & 0.20 & 1.53 & 0.22 & 30 & 4 \\
\hline $\mathrm{B} 1205+1436^{\star}$ & 0.65 & -0.52 & 0.83 & 0.18 & 0.81 & 0.03 & 161 & 6 \\
\hline B1210+1942 & -0.29 & 0.15 & 0.33 & 0.19 & 0.28 & 0.10 & 76 & 19 \\
\hline $\mathrm{B} 1215+1244^{\star}$ & 0.52 & 0.34 & 0.62 & 0.24 & 0.58 & 0.06 & 17 & 12 \\
\hline $\mathrm{B} 1216+1103^{\star}$ & -0.34 & 0.47 & 0.58 & 0.19 & 0.55 & 0.02 & 63 & 10 \\
\hline $\mathrm{B} 1219+1244^{\star}$ & 0.36 & -0.58 & 0.68 & 0.20 & 0.65 & 0.02 & 151 & 9 \\
\hline B1222+1437 & -0.15 & 0.18 & 0.23 & 0.18 & 0.17 & 0.19 & 65 & 30 \\
\hline J $1225-0150^{\star}$ & -0.43 & -0.67 & 0.80 & 0.22 & 0.77 & 0.10 & 119 & 8 \\
\hline $\mathrm{B} 1228+1216^{\star}$ & 0.03 & -0.12 & 0.12 & 0.18 & 0.00 & 0.17 & 142 & - \\
\hline $\mathrm{B} 1230+1705^{\star}$ & -0.28 & -0.11 & 0.30 & 0.19 & 0.25 & 0.05 & 101 & 22 \\
\hline B1230-2347 & -0.04 & 0.03 & 0.05 & 0.19 & 0.00 & 0.57 & 72 & - \\
\hline B1234-0209 & -0.44 & 0.31 & 0.54 & 0.18 & 0.51 & 0.08 & 72 & 10 \\
\hline B1235-1813 & 0.97 & -0.33 & 1.02 & 0.18 & 1.00 & 0.09 & 171 & 5 \\
\hline $\mathrm{B} 1235+1807^{\star}$ & 0.05 & 0.10 & 0.11 & 0.24 & 0.00 & 0.07 & 32 & - \\
\hline B1238-0944 & -0.17 & 0.06 & 0.18 & 0.18 & 0.00 & 0.18 & 80 & - \\
\hline $\mathrm{B} 1239+0955^{\star}$ & 0.65 & -0.50 & 0.82 & 0.18 & 0.80 & 0.00 & 161 & 6 \\
\hline B1239+1435 & 0.13 & 0.12 & 0.18 & 0.20 & 0.00 & 0.05 & 21 & - \\
\hline B1242+0006 & -0.08 & 0.20 & 0.22 & 0.19 & 0.14 & 0.00 & 56 & 39 \\
\hline J $1252+0053^{\star}$ & -0.02 & -0.21 & 0.21 & 0.18 & 0.14 & 0.00 & 132 & 37 \\
\hline B1256-1734 & -0.71 & 0.57 & 0.91 & 0.19 & 0.89 & 0.27 & 71 & 6 \\
\hline B1258-1627 & -0.06 & -0.53 & 0.53 & 0.18 & 0.50 & 0.12 & 132 & 10 \\
\hline B1305+0011 & 0.37 & -0.59 & 0.70 & 0.22 & 0.67 & 0.02 & 151 & 9 \\
\hline $\mathrm{B} 1333+2840^{\star}$ & 4.66 & -3.58 & 5.88 & 0.20 & 5.88 & 0.03 & 161 & 1 \\
\hline $\mathrm{B} 1334+2614^{\star}$ & -0.14 & -0.18 & 0.23 & 0.19 & 0.16 & 0.03 & 116 & 34 \\
\hline B1416-1256 & -0.14 & 0.34 & 0.37 & 0.19 & 0.33 & 0.56 & 56 & 16 \\
\hline B1429-0053 & 0.29 & 0.18 & 0.34 & 0.19 & 0.30 & 0.17 & 16 & 18 \\
\hline B1429-0036* & -0.06 & -0.04 & 0.07 & 0.20 & 0.00 & 0.16 & 107 & - \\
\hline $\mathrm{B} 1443+0141^{\star}$ & 1.00 & -0.87 & 1.33 & 0.23 & 1.31 & 0.27 & 159 & 5 \\
\hline B1451-3735 & 0.11 & -0.24 & 0.26 & 0.18 & 0.21 & 0.56 & 147 & 25 \\
\hline B1500+0824 & -1.09 & -0.38 & 1.15 & 0.33 & 1.10 & 0.08 & 100 & 9 \\
\hline B2118-4303* & -0.04 & -0.66 & 0.66 & 0.20 & 0.63 & 0.16 & 133 & 9 \\
\hline B2149-2745* & -0.13 & 0.07 & 0.15 & 0.20 & 0.00 & 0.14 & 76 & - \\
\hline B2226-3905 & -0.13 & 0.16 & 0.21 & 0.19 & 0.12 & 0.00 & 65 & 45 \\
\hline B2240-3702* & 1.16 & 1.75 & 2.10 & 0.19 & 2.09 & 0.00 & 28 & 3 \\
\hline B2240-3702 & 1.33 & 0.73 & 1.52 & 0.20 & 1.51 & 0.00 & 14 & 4 \\
\hline B2329-3828 & -0.12 & -0.42 & 0.44 & 0.19 & 0.40 & 0.00 & 127 & 14 \\
\hline B2341-2333 & -0.28 & -0.58 & 0.64 & 0.20 & 0.61 & 0.02 & 122 & 9 \\
\hline
\end{tabular}




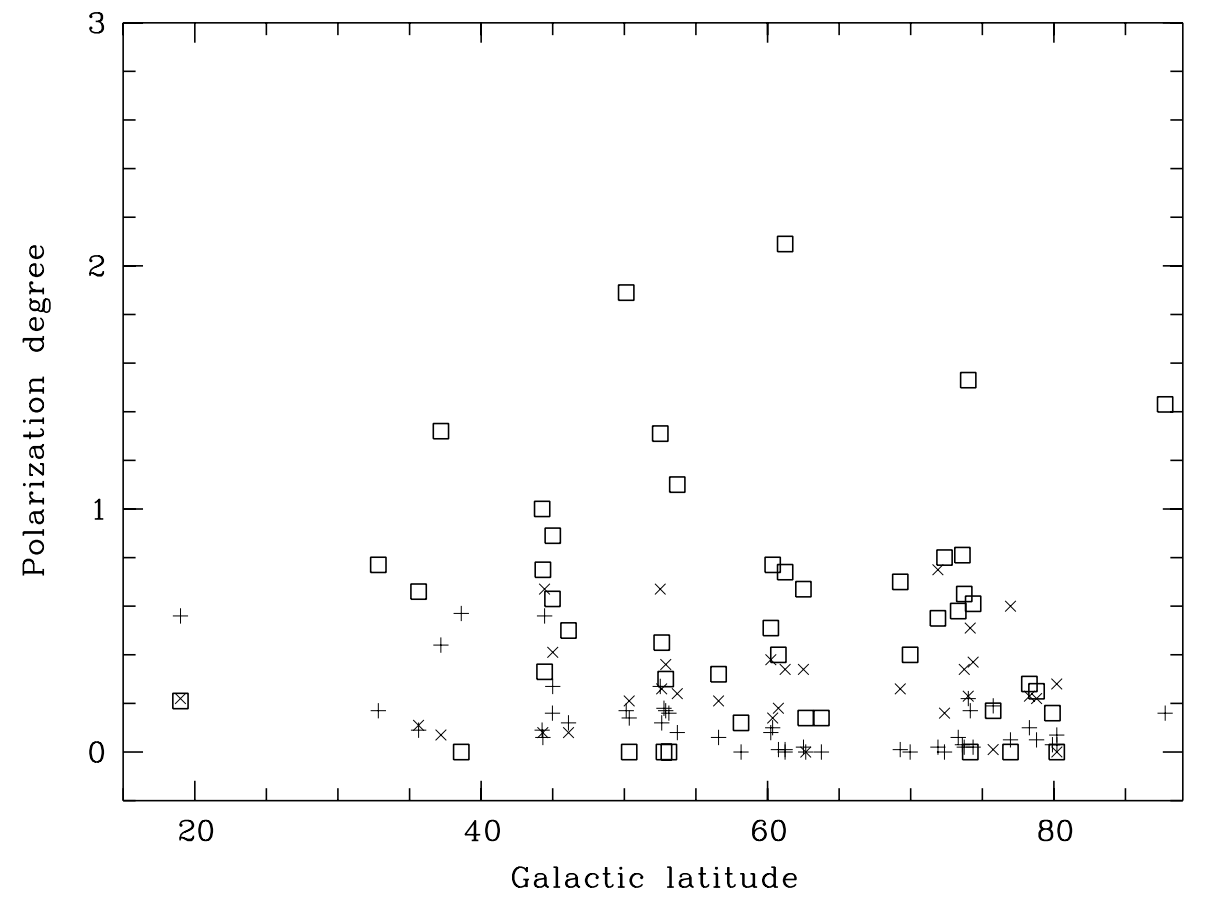

Fig. 1. The QSO polarization degree $p_{0}$ (in \%) [ $\left.\square\right]$ is represented here as a function of the Galactic latitude of the objects $\left(\left|b_{\mathrm{II}}\right|\right.$, in degree), together with the de-biased polarization degree of field stars $[x]$ (also corrected for the small systematic trend reported in Table 2), and the maximum interstellar polarization degree $p_{\text {ISM }}$ derived from the Burstein \& Heiles (1982) reddening maps $[+]$. Only $\mathrm{B} 1333+2840\left(p_{0}=\right.$ $5.9 \%$ ) is not represented here computing the errors on the intensities $I^{\mathrm{u}}$ and $I^{1}$, from the read-out noise and from the photon noise in the object and the sky background (after converting the counts in electrons), and then by propagating these errors in Eqs. (1) or (2). Uncertainties are typically around $0.1 \%$ for both $q$ and $u$.

Since on most CCD frames field stars are simultaneously recorded, one can in principle use them to estimate the residual instrumental polarization, and to correct frame-by-frame the QSO Stokes parameters. However, the field stars (even when combined in a single "big" one per frame) are often fainter than the QSO, and a frame-byframe correction introduces uncertainties on the QSO polarization larger than the instrumental polarization itself. We then computed the weighted average $\left(\bar{q}_{\star}\right.$ and $\left.\bar{u}_{\star}\right)$ and dispersion $\left(\bar{\sigma}_{\star}\right)$ of the normalized Stokes parameters of field stars considering the $\left(n_{\star}\right)$ frames obtained during a given run. These values are given in Table 2 for the two runs with enough data. Note that possible contamination by interstellar polarization is included in the uncertainties. These values indicate that the residual instrumental polarization is small, as expected since most of the instrumental polarization is removed by the observing procedure. We nevertheless take it into account in a rather conservative way by subtracting the systematic $\bar{q}_{\star}$ and $\bar{u}_{\star}$ from the QSO $q$ and $u$, and by adding quadratically the errors. For those objects observed in other runs, no systematic correction was applied; only the errors were similarly combined assuming, quite reasonably, that they are typical of the instrument.

\section{The results}

Table 3 lists the QSO position-name (B1950 or J2000), its redshift $z$, the date of observation (dd-mm-yy), the filter used, the QSO normalized Stokes parameters $q$ and $u$, the uncertainty $\sigma$ of the Stokes parameters ${ }^{2}$, as well as the field star normalized Stokes parameters $q_{\star}$ and $u_{\star}$ and the associated uncertainty $\sigma_{\star}$. The normalized Stokes parameters are given in the equatorial reference frame. Objects marked with an asterisk are BAL QSOs (cf. Brotherton et al. 1998; Green et al. 1997; Korista et al. 1993; Schmidt \& Hines 1999; Stocke et al. 1992; Hewitt \& Burbidge 1993 and references therein).

The final values of the QSO normalized Stokes parameters $q$ and $u$, corrected for the residual systematic instrumental polarization (cf. Table 2) are given in Table 4. Then, from these values, the polarization degree is evaluated with $p=\left(q^{2}+u^{2}\right)^{1 / 2}$. The error on the polarization degree is estimated by $\sigma_{p}=\left(\sigma^{2}+\bar{\sigma}_{\star}^{2}\right)^{1 / 2}$ taking into account the values of Table 2 . Note that the complex statistical behavior of the polarization degree should be kept in mind (Serkowski 1962; Simmons \& Stewart 1985). Indeed, since $p$ is always a positive quantity, it is biased at low signal-to-noise ratio. A reasonably good estimator of the true polarization degree, noted $p_{0}$, is computed from $p$ and $\sigma_{p}$ using the Wardle \& Kronberg (1974) method (Simmons \& Stewart 1985). The polarization position angle $\theta$ is obtained by solving the equations $q=p \cos 2 \theta$ and $u=p \sin 2 \theta$, while the uncertainty of the polarization

\footnotetext{
${ }^{2}$ Note the $u$ Stokes parameter of B1219+1244 may be more uncertain than reported due to a possible contamination of the object image in one of the four frames.
} 
position angle $\theta$ is estimated from the standard Serkowski (1962) formula where $p_{0}$ is used instead of $p$ to avoid biasing, i.e. $\sigma_{\theta}=28.65 \sigma_{p} / p_{0}$. All these quantities are given in Table 4 . Note that due to the HWP chromatism over the $V$ band, an additional error on $\theta \leq 2-3^{\circ}$ should probably be accounted for (cf. the wavelength dependence of the polarization position angle offset in di Serego Alighieri 1998).

Since nearly all objects in the sample are at high galactic latitudes $\left(\left|b_{\mathrm{II}}\right|>30^{\circ}\right.$, except B1451 - 3735), the contamination by interstellar polarization in the Galaxy is expected to be small. This may be verified using the Burstein \& Heiles (1982) reddening maps ${ }^{3}$. The maps provide $E(B-V)$ values from which the interstellar polarization is estimated with the relation $p_{\text {ISM }} \leq 8.3 \% E(B-V)$ (Hiltner 1956). These upper limits on $p_{\text {ISM }}$ are reported in Table 4. All but four are smaller than $0.3 \%$, indicating a negligible contamination by the Galaxy. Polarization of faint field stars recorded on the CCD frames also provide an estimate of the interstellar polarization. The dispersion of their Stokes parameters (Table 2) indicates that actually both the instrumental and interstellar polarization are small. This is illustrated in Fig. 1, where the QSO polarization is compared to the field star polarization (interstellar + instrumental), and to the maximum interstellar polarization derived from the Burstein \& Heiles maps. We may therefore safely conclude that virtually any QSO with $p_{0} \geq 0.6 \%$ is intrinsically polarized, in good agreement with our previous results (Hutsemékers et al. 1998), and with those of Berriman et al. (1990) obtained for lowpolarization Palomar-Green QSOs. Note that several objects with $p_{0}<0.6 \%$ have a polarization position angle similar to that of field stars, indicating that contamination is probably real at these low polarization levels (while no deviation from uniformity is found in the distribution of angle difference for objects with $p_{0} \geq 0.6 \%$ ).

For some QSOs of our sample (B0059 - 2735, B1157 2354, B1429 - 0053, B2240 - 3702), polarimetric measurements have been obtained at different epochs with the same filter and instrumentation (cf. Hutsemékers et al. 1998). The agreement is generally excellent, providing no evidence for polarization variability. Only the polarization degree of B1429 - 0053 is marginally different, possibly in relation with its suspected gravitationally lensed nature.

\section{Conclusions}

New broad-band linear polarization measurements have been obtained for a sample of 47 QSOs down to an accuracy of $\sim 0.2 \%$. Most data are first-time measurements. The sample includes 27 BAL QSOs and 2 gravitational lens candidates (B1429 - 0053 and B2149 - 2745). With previous surveys by
Hutsemékers et al. (1998) and Schmidt \& Hines (1999), approximately 70 BAL QSOs have now measured polarization.

The present data show little contamination by interstellar polarization, and virtually any QSO with $p \geq 0.6 \%$ is intrinsically polarized. Nine objects have $p \geq 1.0 \%$, and one BAL QSO, B1333+2840, has $p=5.9 \%$. No polarization variability is detected, except, possibly, for the gravitational lens candidate B1429 - 0053.

Acknowledgements. It is a pleasure to thank Ingunn Burud for her participation in the September 99 observations, and Pierre Leisy for efficient support. This research is supported in part by contracts ARC 94/99-178 and PAI P4/05.

\section{References}

Berriman G., Schmidt G.D., West S.C., Stockman H.S., 1990, ApJS 74, 869

Brotherton M.S., van Breugel W., Smith R.J., et al., 1998, ApJ $505, \mathrm{~L} 7$

Burstein D., Heiles C., 1982, AJ 87, 1165 (BH)

di Serego Alighieri S., 1989, in $1^{\text {st }}$ ESO/ST-ECF Data Analysis Workshop, Grosbøl P.J. et al. (eds.), p. 157

di Serego Alighieri S., 1998, in Instrumentation for Large Telescopes, Rodriguez Espinosa J.M. (ed.). Cambridge University Press, p. 287

Green P.J., Aldcroft T.L., Mathur S., Schartel N., 1997, ApJ 484, 135

Hewitt A., Burbidge G., 1993, ApJS 87, 451

Hiltner W.A., 1956, ApJS 2, 389

Hutsemékers D., 1998, A\&A 332, 410

Hutsemékers D., Lamy H., Remy M., 1998, A\&A 340, 371

Lamy H., Hutsemékers D., 1999, The Messenger 96, 25 (Erratum: The Messenger 97, 23)

Korista K.T., Voit G.M., Morris S.L., Weymann R.J., 1993, ApJS 88, 357

Melnick J., Dekker H., D'Odorico S., 1989, EFOSC, ESO operating manual No. 4, Version 2, ESO

Moore R.L., Stockman H.S., 1981, ApJ 243, 60

Moore R.L., Stockman H.S., 1984, ApJ 279, 465

Patat F., 1999, EFOSC2 user's manual, Version 1, ESO

Schmidt G.D., Elston R., Lupie O.L., 1992, AJ 104, 1563

Schmidt G.D., Hines D.C., 1999, ApJ 512, 125

Schwarz H.E., 1987, in Polarimetry with EFOSC, ESO Internal Memorandum Dec. 1987

Schwarz H.E., Guisard D., 1995, The Messenger 81, 9

Serkowski K., 1962, in Advances in Astron. and Astrophys., Kopal Z. (ed.). Academic Press 1, p. 289

Simmons J.F.L., Stewart B.G., 1985, A\&A 142, 100

Stocke J.T., Morris S.L., Weymann R.J., Foltz C.B., 1992, ApJ 396,487

Turnshek D.A., Bohlin R.C., Williamson II R.L., Lupie O.L., Koornneef J., Morgan D.H., 1990, AJ 99, 1243

Wardle J.F.C., Kronberg P.P., 1974, ApJ 194, 249

Whittet D.C.B., Martin P.G., Hough J.H., Rouse M.F., Bailey J.A., Axon D.J., 1992, ApJ 386, 562

\footnotetext{
3 The data files and routines were obtained from Schlegel 1998, via http://astro.berkeley.edu/davis/dust/data/bh/index.html
} 